\title{
«Paralipomena» on uniqueness in inverse scattering from a finite number of data
}

\author{
Raffaele Persico \\ Istituto per i Beni Archeologici e Monumentali (IBAM), CNR, Lecce, Italy
}

\begin{abstract}
This paper shows new proof of non-uniqueness of the solution for the retrieving of a compact-supported function starting from a finite number of samples of its spectrum. As will be shown, this is relevant for linear inverse scattering problems, that in many cases can be recast as the reconstruction of a compact supported function from a finite set of samples of its spectrum. Since this reconstruction is not unique, from a practical point of view, any linear inverse scattering algorithm that can be recast in terms of a Fourier relationship between unknowns and data necessarily «trusts» on the absence of invisible objects in the particular situation at hand.
\end{abstract}

Key words Fourier transform - diffraction tomography - inverse scattering

\section{Introduction}

The reconstruction of a compact-supported function starting from a finite number of samples of its spectrum is a problem potentially of interest in various fields. In particular, inverse scattering problems in a loss-less background medium can be often recast as the reconstruction of some compact supported object function from a limited portion of its spectrum (Lesselier and Duchene, 1996; Persico and Soldovieri, 2004a). Due to the regularity of the spectrum of a compactsupported (square-integrable) function, in some cases there is a theoretical possibility to prolong this spectrum outside the limited region directly related to the data (Slepian and Pollack, 1961) (apart from ill-posedness problems, not explicit-

Mailing address: Dr. Raffaele Persico, Istituto per i Beni Archeologici e Monumentali (IBAM), CNR, Via Monteroni, Campus Universitario, 73100 Lecce, Italy; e-mail: r.persico@ibam.cnr.it ly considered here). This rationale has given rise to various uniqueness theorems for the solution of several inverse problems (Colton and Paivarinta, 1992; Sheen and Shepelsky, 2000). However, uniqueness theorems always require a continuous set of data to be available and often assume some regularity of the object function, which is not achieved in many practical cases (Ramm, 1990; Colton and Paivarinta, 1992). Moreover, and above all, this regularity cannot usually be supposed a priori because, let us remember, the object function is the unknown of the problem. Finally, even if the object function were regular (i.e. continuous with its spatial derivatives of the first order) and a continuous (multi-experiment) set of data were available, still the uniqueness might not be guaranteed in some cases (Devaney, 1978; Fisher and Langenberg, 1984).

If the number of data is finite, instead, it is just impossible to retrieve a compact-supported function from a finite number of samples of its Fourier Transform uniquely. It is opinion of the author that this fact is worth outlining at least once, because in any practical case one has at disposal necessarily only a finite number of data. The above mentioned impossibility stems from the fact that a compact-supported object function is isomorphic to a countable set of ex- 
pansion coefficients along some functional basis. This means that the object function is characterised by an infinite number of independent parameters, that cannot correspond in a biunique fashion to a finite number of data. The only way to solve this kind of inverse problem uniquely is to choose a suitable finite dimensional space wherein to look for the solution. In this way, a whole class of somewhat «improbable» solutions is discarded a priori.

In spite of the simplicity of these considerations, however, it is not immediate to show explicitly (i.e. by providing an example) the nonuniqueness of the solution of the problem at hand, so that one could forget the fact that, when considering a finite number of data, the solution to any inverse scattering problem is actually only one of the possible solutions. From a practical point of view this means, for example, that when one performs the reconstruction of the inner of a subsurface region by elaborating GPR (Alberti et al., 2002, 2003; Daniels, 2004) data, they can never be mathematically sure of their reconstruction results. They can say that those results are reasonable and probable but, from a mathematical point of view, they cannot exclude the possibility that the actual buried objects are something strange and complicated, even very different from the foreseen objects but compatible with the same gathered data. Finally, they should not ascribe this possibility only to the presence of some (even possible) non-radiating density of current or to the lack of conditions for the validity of some complicated uniqueness theorem. Rather, they should recognize the much more immediate fact that they necessarily have only a finite number of data available. This paper is aimed to show this fact explicitly. More precisely, the pursued goal is to show, by means of a counterexample, the fact that the reconstruction of a compact supported function from a finite number of samples of its Fourier Transform is never unique.

Actually, the result demonstrated here is already known (Levinson, 1940; Levin, 1980). However, to show a systematic way to obtain counterexamples is, to the best of the author's knowledge, a new result. Moreover, apart from the «novelty» of the approach to this «old» problem, the effort presented here seemed worth making because the new proof is quite simple, in the sense that it does not require a deep knowledge of the properties of the entire functions (Levin, 1980) in order to be understood. In particular, it is plainly accessible for people that have a non-trivial knowledge of mathematics (as, e.g., geophysicists and engineers), but nevertheless are not mathematicians.

The next section provides some examples of physical inverse problems that can be recast as the reconstruction of a compact supported function from a finite number of spectral data. In this way, the physical sense of the mathematical problem dealt with here will be more precisely circumstantiated. Then, in Section 3 a method to construct the sought counterexample is exposed. An example is provided in Section 4. Conclusions follow.

\section{Spectral algebraic relationships in inverse problems}

This section provides some algebraic relationships connecting the available scattered field data to the object function to be reconstructed in microwave inverse problems. These relationships are picked up from that branch of electromagnetic inverse problems commonly referred to as Diffraction Tomography (DT) (Lesselier and Duchene, 1996), and the aim of this exposition is to give a physical sense to the (otherwise merely mathematical) dealing of the two next sections. In this paper, DT will be somewhat prompted and essentially referred. Of course, this brief report does not claim to be a review and is, necessarily, far from complete.

After this premise, in DT the problem is to retrieve buried objects, or more in general buried inhomogeneities, from scattered field data. The general three dimensional geometry of the problem is given in fig. 1, wherein the data are constituted by samples of the scattered field gathered within the observation domain $\Gamma$ at several frequencies within a fixed band $\Omega$, and the buried objects are described by means of the so called contrast function, defined as

$$
\chi(P)=\left\{\begin{array}{l}
\frac{\varepsilon_{r}(P)}{\varepsilon_{\text {bkg }}}-1 \quad P \in D \\
0 \text { elsewhere }
\end{array}\right.
$$




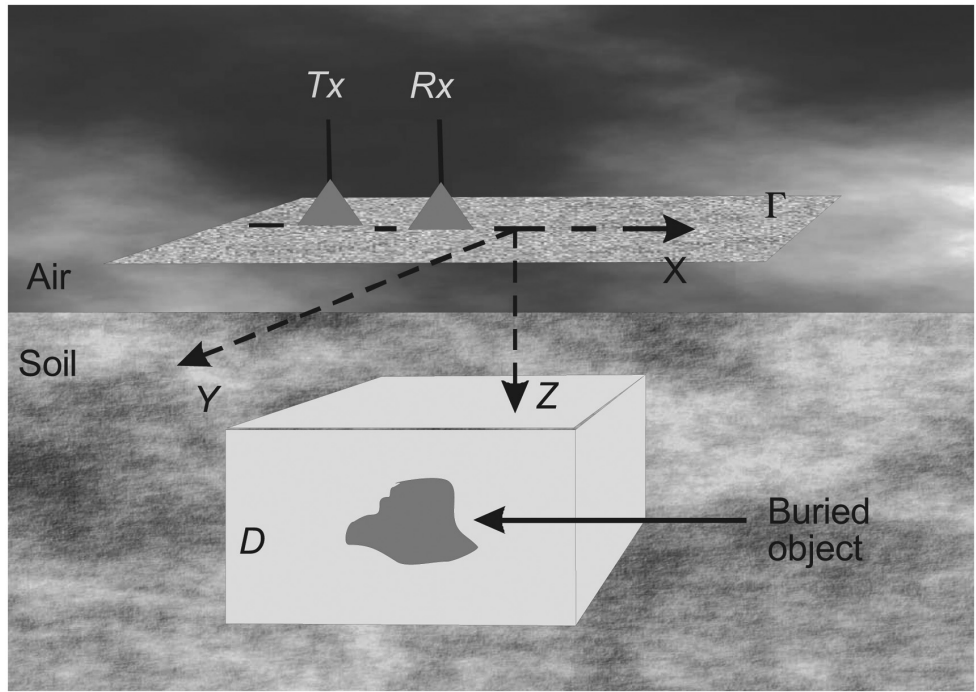

Fig. 1. Geometry of the problem.

where $\varepsilon_{r}(P)$ is the unknown relative dielectric permittivity in the investigation domain $D$ and $\varepsilon_{\text {bkg }}$ is the relative background permittivity, i.e. the relative dielectric permittivity of the soil (or of the layered medium) where the object is buried. From eq. (2.1), we can see that the contrast expresses the relative difference between the dielectric permittivity of the buried inhomogeneities and that of the host medium surrounding them. In general, the contrast is also a function of the frequency, but this fact is often neglected in the adopted model, unless a dispersion law is a priori assumed (Catapano et al., 2006). Under a linear Born model (Chew, 1995), and with reference to fig. 1, the relationship between the contrast and the scattered field data is given by (Lesselier and Duchene, 1996)

$$
\begin{aligned}
& E_{s}\left(P_{o}, P_{s}, \omega\right)=k_{s}^{2} \int_{D} G\left(P_{o}, P^{\prime}, \omega\right) E_{\text {inc }}\left(P_{s}, P^{\prime}, \omega\right) \chi\left(P^{\prime}\right) \mathrm{d} P^{\prime} \\
& \omega \in \Omega, P_{s}, P_{o} \in \Gamma
\end{aligned}
$$

where $G$ is Green's function of the problem, $E_{\text {inc }}$ is the incident field (i.e. the field that would be present in the investigation domain $D$ in absence of any buried object), $k_{s}=\omega \sqrt{\varepsilon_{\text {bkg }}} / c_{0}$ is the wavenumber in the background medium (being $c_{0}$ the propagation speed of the light in free space), $P_{o}$ is the observation point and $P_{s}$ is the source point, both supposed variable within the observation domain $\Gamma$ and, finally, $P^{\prime}$ is the integration point variable in the investigation domain $D$. If losses can be neglected, several spectral relationships can be obtained by taking into consideration particular cases of eq. (2.2). Some of them are provided in the following.

\subsection{The case of a one dimensional half-space}

The case of a one dimensional half-space can be of interest (e.g.) when a humidity profile or any stratification profile only varying versus the depth is looked for. If the source is constituted by a plane wave impinging from the upper half-space, the inverse problem can be recast as a one dimensional scalar problem wherein a contrast $\chi(z)$ is looked for. In this case, it can be shown that the only independent observation parameter is the frequency, whereas some fur- 
ther independent source parameters can be provided by diversity in incidence angle. For more details the reader is referred to Pierri et al. (1999), Persico and Soldovieri (2004a) and, Soldovieri and Persico (2004). Here, we limit to the case of Transverse ElectroMagnetic (TEM) incidence. In this case, the substitution of the specific values of Green's function and of the incident field in eq. (2.2) provides, after some passages (Soldovieri and Persico, 2004)

$$
E_{s}(\omega)=g(\omega) \hat{\chi}\left(2 k_{s}\right) \quad \omega \in \Omega
$$

where $g(\omega)=\left[\left(-j 2 k_{o} k_{s} E_{o}\right) /\left(k_{o}+k_{s}\right)^{2}\right] k_{s}$, being $E_{o}$ the amplitude of the impinging plane wave and $k_{0}=\omega / c_{0}$ the wavenumber in free space. In eq. (2.3), and also in the following equations, the hat symbols stand for «Fourier Transform».

From eq. (2.3), it is clear that gathering scattered field data at $N$ frequencies $\omega_{1} \ldots \omega_{N}$ corresponds to retrieving the spectrum of the contrast function in a finite set of points $2 k_{s 1} \ldots 2 k_{s N}$. Moreover the contrast is compact supported because the investigation domain necessarily extends only up to a certain finite depth.

\subsection{The case of a two dimensional half-space}

The case of a two dimensional half-space is of scientific interest for retrieving elongated objects (e.g., buried pipes or buried walls). Moreover, a two dimensional model is of applicative interest in many three dimensional situations, because it often offers acceptable results in a much shorter time with respect to a three dimensional algorithm. In a two dimensional half-space, the object function is variable along the depth $z$ and along a horizontal abscissa $x$ (but it is constant along the other horizontal direction $y$ ). If the incident field is provided by a filamentary current parallel to the $y$-axis, the inverse problem can be recast as a two dimensional scalar problem. In particular, limiting to the case of two antennas that move on the soil along the $x$-axis with a fixed offset $\Delta$ between each other (which corresponds to the so called common offset configuration, customarily adopted in GPR prospecting; Daniels, 2004), substitution of the proper values of Green's function and of the incident field in eq. (2.2) provides, after some passages, the relationship

$$
\hat{E}_{s}(p)=g(p) \hat{\hat{\chi}}_{1}(\eta(p), s(p))
$$

where

$$
\begin{gathered}
g(p)=\frac{j k_{s}^{2} f I_{o} \mu_{o} \exp \left(j\left(\frac{\pi}{4}+\frac{p \Delta}{2}\right)\right)}{\left(w_{o}\left(\frac{p}{4}\right)+w_{s}\left(\frac{p}{2}\right)\right)^{2}} . \\
\sqrt{\frac{\pi\left(w_{2}\left(\frac{p}{4}\right)\right)^{3}}{\left(\frac{p}{2}\right)^{2}+\left(w_{2}\left(\frac{p}{2}\right)\right)^{2}}} \\
\left\{\begin{array}{l}
w_{o}(p)=\sqrt{k_{0}^{2}-p^{2}} \\
w_{s}(p)=s \sqrt{k_{s}^{2}-p^{2}}
\end{array}\right.
\end{gathered}
$$

and where

$$
\left\{\begin{array}{l}
\eta(p)=p \\
s(p)=2 w_{s}\left(\frac{p}{2}\right)=\sqrt{4 k_{s}^{2}-p^{2}} .
\end{array}\right.
$$

In eqs. ((2.4)-(2.6)), $p$ is the conjugate variable of the source position $x_{s}$ (the observation position is $x_{s}+\Delta$ ), whereas $\eta$ and $\xi$ are the conjugate variables of the horizontal abscissa $x$ and of the depth $z$ in the investigation domain, respectively. The object function is $\chi_{1}(x, z)=\chi(x, z) / \sqrt{z}$. The quantities in eq. (2.4) also depend on the frequency, that appears both in the spectral weighting function $g(p)$ and (above all) in the arguments of the spectrum of the contrast (see eq. (2.6)). At the right-hand side of eq. (2.5), $f$ is the frequency, $\mu_{o}$ is the magnetic permeability of the free space and $I_{o}$ is the level of the current. Unlike the previously shown one dimensional situation, in this case we do not have a direct proportionality between the data and the spectrum of the object function, but rather we have proportionality between the spectrum of the data and the spectrum of the object function. This is due to the fact that in two dimensional problems we can extract information from spatial diversity of the observation point, which could not been done in one dimensional cases. From a practical point of view, however, this does not change the problem too much. In fact, on condition that the error 
due to the truncation of the scattered field along $x_{s}$ can be neglected (which is reasonable when the source moves far from the buried objects), from a finite number of samples of the scattered field one can work out a finite number of independent samples of the spectrum of the scattered field by means of an FFT algorithm. At this point, eq. (2.4) relates these samples of the spectrum of the scattered field to the corresponding samples of the spectrum of an object function linked to the contrast. In conclusion, also in this case, what we can retrieve from a finite number of data is essentially a finite number of samples of the spectrum of a proper object function. For more details, the interested reader is referred to Persico et al. (2005).

\subsection{The case of a three dimensional half-space}

In the case of a fully three dimensional halfspace the mathematics is more involved, and it is somewhat foreseeable. Essentially, the point is that in three dimensions we cannot avoid the intrinsic vector aspects of the problem. In particular, Green's function of eq. (2.2) has to be considered a dyadic one, and we should understand the product between Green's function and the incident field as a matrix-vector product. In formulas we have

$$
G=\left(\begin{array}{lll}
G_{x x} & G_{x y} & G_{x z} \\
G_{y x} & G_{y y} & G_{y z} \\
G_{z x} & G_{z y} & G_{z z}
\end{array}\right) \quad E_{\text {inc }}=\left(\begin{array}{c}
E_{\text {incx }} \\
E_{\text {incy }} \\
E_{\text {incz }}
\end{array}\right) .
$$

Equation (2.7) means that each of the components of the incident field influences all the components of the scattered field. In the following, the attention is focussed on Hertzian dipole antennas placed on a soil parallel to each other along the $y$ axis and moved in common offset along several lines parallel to the $x$ axis. This is a case that describes quite well many GPR prospecting operations performed with linearly polarized antennas (Daniels, 2004). In this case, we essentially gather a signal proportional to the $y$ component of the scattered field and therefore, one more time, we can refer to a scalar inverse problem recast as

$$
\begin{aligned}
& E_{s y}\left(P_{o}, P_{s}, \omega\right)=k_{s}^{2} \int_{D} K r\left(P_{o}, P_{s}, P^{\prime}, \omega\right) \chi\left(P^{\prime}\right) \mathrm{d} P^{\prime} \\
& \omega \in \Omega, P_{s}, P_{o} \in \Gamma
\end{aligned}
$$

where the kernel of the integral operator is defined as

$$
\operatorname{Kr}\left(P_{o}, P_{s}, P^{\prime}, \omega\right)=G_{y x} E_{\text {incx }}+G_{y y} E_{\text {incy }}+G_{y z} E_{\text {incz }} .
$$

A substitution of the proper values of the incident field and of the Green's function in eq. (2.8) leads to a spectral relationship of the following kind:

$$
\hat{\hat{E}}_{s y}(u, v)=g(u, v) \hat{\hat{\chi}}_{2}(\eta(u, v), \xi(u, v), \zeta(u, v)) .
$$

where

$$
\left\{\begin{array}{l}
\eta(u, v)=u \\
\xi(u, v)=v \\
\zeta(u, v)=\sqrt{4 k_{s}^{2}-u^{2}-v^{2}}
\end{array}\right.
$$

In eqs. ((2.10)-(2.11)), $u$ is the conjugate variable of the first coordinative of the source position $x_{s}, v$ is the conjugate variable of the second coordinative of the source position $y_{s}$ (the observation position in this case is given by $\left(x_{s}+\Delta\right.$, $\left.\left.y_{s}\right)\right), \eta$ and $\xi$ are the conjugate variables of the horizontal co-ordinates $x$ and $y$ and $\zeta$ is the conjugate variable of the depth $z$ in the investigation domain, respectively. The object function is defined as $\chi_{2}(x, y, z)=\chi(x, y, z) / z$. It can be seen that eq. (2.10) is a quite «natural» extension of eq. (2.4). With respect to eq. (2.4), we have a further spectral variable with regard to the scattered field (because the observation domain is a surface rather than a line) and a further spectral variable with regard to the contrast (because the investigation domain is a buried volume rather than a buried area). For more details, and in particular for the explicit expression of the spectral weighting function $g(u, v)$, the interested reader is referred to Hansen and Johansen (2000). What is relevant here is to outline that, by means of a two dimensional FFT, also in this case a finite number of sam- 
ples of the scattered field essentially provides a finite number of samples of the spectrum of the object function. Therefore, one more time the problem can be recast as the reconstruction of the object function $\chi_{2}$, compact supported in the investigation domain $D$, from a finite number of samples of its spectrum.

\subsection{Prompts on further relevant cases}

Several further possible cases of interest can be recast as the retrieving of some object function from a finite number of samples of its spectrum. The essential reason for this fact is that, in eq. (2.2), both Green's function and the incident field can be expressed by means of their Plane Wave Spectrum (PSW) (Clemmow, 1996). Due to this fact, the problem of retrieving inhomogeneities buried in any lossless layered medium (i.e. not only half-spaces) can be dealt with by making use of suitable DT spectral algebraic relationships. In particular, the case of a one dimensional slab (i.e. a one dimensional medium with three layers) has been dealt in Persico and Soldovieri (2004a,b), whereas the case of a two dimensional slab has been dealt with in Crocco and Soldovieri (2003). Furthermore, also the radiation characteristics of the transmitting and receiving antennas can be included in a DT formulation. Essentially, the radiation characteristics of the antennas affect the spectral weighting function $g$. A two dimensional dealing of this problem can be found in Soldovieri et al. (2005a), whereas a three dimensional dealing can be found in Meincke (2001). Still, different configurations (other than common offset) can also be dealt with by means of a DT formalism. Essentially, in these cases the arguments of the spectrum of the object function will be somewhat varied. For more details, the interested reader is firstly referred to Persico et al. (2005), and then also to Lesselier and Duchene (1996) and Soldovieri et al. (2005a,b). Finally, even some aspects relative to the consequences of the truncation of the observation domain can be understood in terms of a DT formulation. Details can be found in Leone and Soldovieri (2003) and Persico et al. (2006).

\section{A method to construct a counterexample}

In this section we come back to the mathematical problem presented in the introduction. In particular, this section is devoted to finding a (non null) function with a compact support such as its spectrum is equal to zero in a finite set of points $k_{1}, k_{2}, \ldots, k_{N}$. In particular, the problem is afforded in the one dimensional case for the sake of simplicity in the exposition, but extensions to more complicated cases (two and three dimensional) are straightforward. Of course, such a function is an element of the null space of the linear operator that transforms a compact supported function into the column vector of the values of its spectrum in the points $k_{1}, k_{2}, \ldots, k_{N}$. Therefore, to find such a function means to prove that the (semi-discrete) operator at hand can never be univocally inverted (Bertero and Boccacci, 1998).

The searched function can be found as the limit of a succession of functions, i.e. it can be built. In order to initialize the algorithm, let $\chi_{1}(z)$ be an arbitrary square integrable function supported in prefixed interval $[-a, a]$, i.e. be $\chi_{1} \in L^{2}(-a, a)$.

Let $F_{1}(k)$ be the Fourier transform of $\chi_{1}(z)$. As it is well known, $F_{1}(k)$ is a square integrable function $\left(F_{1} \in L^{2}(R)\right)$ and has relevant regularity properties (Paley and Wiener, 1934). For the purposes of this paper, at any rate, it is sufficient to outline the only fact that $F_{1}(k)$ is continuous (which is well known and, even if it were not known, it could be plainly proved directly).

Let us label $a_{1}, a_{2}, \ldots, a_{N}$ the vector of the values assumed by $F_{1}(k)$ in the points $k_{1}, k_{2}, \ldots$, $k_{N}$ respectively. At this point, let us define a «corrected version of $F_{1}$ » as follows:

$$
\begin{aligned}
& F_{2}(k)=F_{1}(k)-a_{1} \Lambda\left(\frac{k-k_{1}}{M}\right)-a_{2} \Lambda\left(\frac{k-k_{2}}{M}\right)-\ldots \\
& \ldots-a_{N} \Lambda\left(\frac{k-k_{N}}{M}\right)
\end{aligned}
$$

$\Lambda(k)$ being the well known roof function defined as

$$
\Lambda(k)=\left\{\begin{array}{l}
1-|k| \quad k \in(-1,1) \\
0 \text { elsewhere }
\end{array}\right.
$$


$F_{2}$ is continuous because it is a linear combination of continuous functions. Moreover, on condition that $M$ is chosen small enough, we can impose that $F_{2}(k)=0$ in each of the points $k_{1}, k_{2}, \ldots$, $k_{N}$. Moreover, for any chosen positive $K$, on condition that $M$ is chosen small enough we can also guarantee that

$$
\left\|F_{1}-F_{2}\right\| \leq \frac{1}{K}
$$

In particular, we require here that $K>1$. In the end, $F_{2}$ is a slight modification of $F_{1}$ (as close as we like to $F_{1}$ ) that shows the required roots. However, in general $F_{2}$ is not the spectrum of a compact supported function, and therefore the searched result has not been reached yet. Therefore, let $\chi_{2}(z)$ be the inverse Fourier Transform of $F_{2}(k)$, and $\chi_{3}(z)$ be the truncated version of $\chi_{2}(z)$ in the interval $[-a, a]$, i.e. $\chi_{3}$ be defined as

$$
\chi_{3}(z)=\left\{\begin{array}{l}
\chi_{2}(z) \quad z \in[-a, a] \\
0 \quad \text { elsewhere }
\end{array} .\right.
$$

Let us now outline two inequalities. First, since the Fourier transform is an isometry (Kantorovic and Akilov, 1982), we have

$$
\left\|\chi_{1}-\chi_{2}\right\|=\left\|F_{1}-F_{2}\right\| \leq \frac{1}{K} .
$$

Second, as an easy consequence of the definition of $\chi_{3}(z)$ we have

$$
\left\|\chi_{1}-\chi_{3}\right\| \leq\left\|\chi_{1}-\chi_{2}\right\| \leq \frac{1}{K}
$$

In fact, reminding that both $\chi_{1}$ and $\chi_{3}$ have compact support included in $[-a, a]$, it results

$$
\begin{aligned}
& \left\|\chi_{1}-\chi_{2}\right\|^{2}=\int_{-a}^{a}\left|\chi_{1}-\chi_{2}\right|^{2} \mathrm{~d} z+\int_{z \notin[-a, a]}\left|\chi_{2}\right|^{2} \mathrm{~d} z= \\
& \int_{-a}^{a}\left|\chi_{1}-\chi_{3}\right|^{2} \mathrm{~d} z+\int_{z \notin[-a, a]}\left|\chi_{2}\right|^{2} \mathrm{~d} z \geq \int_{-a}^{a}\left|\chi_{1}-\chi_{3}\right|^{2} \mathrm{~d} z= \\
& \left\|\chi_{1}-\chi_{3}\right\|^{2} .
\end{aligned}
$$

Therefore, due to the first triangular inequality (Kantorovic and Akilov, 1982)

$$
\left\|\chi_{2}-\chi_{3}\right\| \leq\left\|\chi_{1}-\chi_{2}\right\|+\left\|\chi_{1}-\chi_{3}\right\| \leq \frac{2}{K}
$$

At this point, let $F_{3}(k)$ be the Fourier transform of $\chi_{3}(k) . F_{3}(k)$ is a continuous function because it is the spectrum of a compact supported square integrable function. Moreover, since the Fourier transform is isometric, we have

$$
\left\|F_{2}-F_{3}\right\|=\left\|\chi_{2}-\chi_{3}\right\| \leq \frac{2}{K} .
$$

Let $F_{4}(k)$ be a «corrected version of $F_{3}(k) »$ constructed in the same fashion in which $F_{2}(k)$ had been built starting from $F_{1}(k)$, so that $F_{4}(k)$ is equal to zero in the points $k_{1}, k_{2}, \ldots, k_{N}$. Moreover, this time we require a new value for $M$, such as it is guaranteed

$$
\left\|F_{3}-F_{4}\right\| \leq \frac{1}{K^{2}}
$$

Of course, we can do this because the choice of $M$ is arbirtary.

Going on, $\chi_{4}(k)$ is the inverse Fourier transform of $F_{4}(k)$ and the cycle begins again. The procedure is iterated by subsequent truncations in the space domain and subsequent «corrections» in the spatial frequency domain. For each even value of the index $w$, a correction in the «k-domain» occurs, and it is imposed that

$$
\left\|F_{w}-F_{w-1}\right\| \leq \frac{1}{K^{0.5 w}} .
$$

Consequently, for each even value of $w$, it results

$$
\left\|\chi_{w}-\chi_{w-1}\right\| \leq \frac{1}{K^{0.5 w}} .
$$

For each odd value of $w$, a truncation in the «zdomain» occurs. The truncation guarantees that, for each odd value of $w$, it results

$$
\left\|\chi_{w}-\chi_{w-1}\right\| \leq \frac{2}{K^{0.5(w-1)}}
$$

and, consequently, it also results

$$
\left\|F_{w}-F_{w-1}\right\| \leq \frac{2}{K^{0.5(w-1)}} .
$$

In this way, two sequences of functions $\chi_{1}, \chi_{2}$, $\ldots, \chi_{n}, \ldots$ and $F_{1}, F_{2}, \ldots, F_{n}, \ldots$ are identified. 
From eqs. ((3.11)-(3.14)), both these successions verify the Cauchy condition (let us remind that we have chosen $K>1$ ), i.e. the condition that the quantity $\left\|\chi_{w}-\chi_{w-1}\right\|=\left\|F_{w}-F_{w-1}\right\|$ vanishes versus the index $w$. Therefore, due to the completeness of the space $L^{2}$, two limit functions $\chi_{L}$ and $F_{L}$ exist for the two successions $\chi_{1}, \chi_{2}, \ldots, \chi_{n}, \ldots$ and $F_{1}, F_{2}, \ldots, F_{n}, \ldots$ respectively (Kantorovic and Akilov, 1982). Moreover, $F_{L}$ is the Fourier transform of $\chi_{L}$ because of the continuity of the Fourier operator.

The sought function is $\chi_{L}$. In fact, $\chi_{L}$ is a function supported within the interval $[-a, a]$ because it is definitively closer and closer to any one of the truncated (compact supported) functions $\chi_{2 w-1}$, on condition that $w$ is high enough. Moreover, the spectrum of $\chi_{L}$ assumes the value zero in the points $k_{1}, k_{2}, \ldots, k_{N}$. In order to show this fact, let us outline that $F_{L}$ is a regular (in particular continuous) function, because it is the Fourier transform of a compact supported function. Therefore, we have that $F_{1}, F_{2}, \ldots, F_{n}, \ldots$ is a succession of continuous functions that converges towards a continuous limit. In these conditions, it is easy to recognize that the convergence in $L^{2}$ also guarantees the punctual convergence, even if it does not in general (Kantorovic and Akilov, 1982).

In order to complete the proof, however, we still have to guarantee that $\chi_{L}$ is not a null function, otherwise only a trivial result would have been found. In order to show this fact, let us note that, due to the first triangular inequality

$$
\begin{aligned}
& \left\|\chi_{1}-\chi_{L}\right\| \leq\left\|\chi_{1}-\chi_{2}\right\|+\left\|\chi_{2}-\chi_{3}\right\|+\ldots\left\|\chi_{w}-\chi_{w-1}\right\|+ \\
& +\ldots \leq \frac{1}{K}+\frac{2}{K}+\frac{1}{K^{2}}+\frac{2}{K^{2}}+\ldots+\frac{1}{K^{n}}+\frac{2}{K^{n}}+\ldots= \\
& =\frac{3}{K}+\frac{3}{K^{2}}+\ldots \frac{3}{K^{n}}+\ldots=3\left(\frac{K}{K-1}-1\right)=\frac{3}{K-1} .
\end{aligned}
$$

In this last passage we have exploited again the hypothesis $K>1$, which guarantees the convergence of the geometrical series of ratio $1 / K$. Inequality (3.15) essentially means that the limit function can be found as close as we like to the initial function $\chi_{1}$ (in other words, the null space of the semi-discrete operator at hand is dense in $\left.L^{2}(-a, a)\right)$. Due to the arbitrary choice of $\chi_{1}$, therefore, it is easy to guarantee that $\chi_{L}$ is a non null function. For example, one can require that

$$
\left\|\chi_{1}\right\| \geq A+\frac{3}{K-1}
$$

$A$ being some positive constant. Consequently, by virtue of the second triangular inequality (Kantorovic and Akilov, 1982), we have

$$
\begin{aligned}
& \left\|\chi_{L}\right\|=\left\|\chi_{L}+\chi_{1}-\chi_{1}\right\| \geq\left|\left\|\chi_{1}\right\|-\left\|\chi_{L}-\chi_{1}\right\|\right|= \\
& \left\|\chi_{1}\right\|-\left\|\chi_{L}-\chi_{1}\right\| \geq A>0
\end{aligned}
$$

and this completes the proof.

\section{An example}

This section illustrates the method for the construction of a compact supported function whose spectrum shows some (imposed) roots, exposed in the previous section, in a particular case.

Therefore, let us suppose that we have to identify a function supported in the interval $[-1,1]$ on the $z$-axis, and let us suppose that we require that the spectrum of this function has to assume the value zero in the points $-0.7 \pi$ $(\cong-2.199)$ and $0.7 \pi(\cong+2.199)$ on the $k$-axis. The initial point is chosen equal to the function

$$
\chi_{1}(z)=\Pi\left(\frac{z}{2}\right)= \begin{cases}1 & z \in[-1,1] \\ 0 & \text { elsewhere }\end{cases}
$$

therefore, the «first function» in the spectral domain is given by

$$
F_{2}(k)=2 \operatorname{sinc}(k)
$$

which shows the first roots at $k= \pm \pi$ (here, $\operatorname{sinc}(k)$ is meant as $\sin (k) / k)$. According to the procedure explained in the previous section, let us choose $K=1.2$, and let us specify a corrected version of $F_{1}$ which shows the required roots. Based on eq. 12 and on the parity of the sinc function, $F_{2}$ can be chosen as

$$
\begin{aligned}
& F_{2}(k)=F_{1}(k)-2 \operatorname{sinc}(0.7 \pi)\left[\Lambda\left(\frac{k-0.7 \pi}{M_{1}}\right)+\right. \\
& \left.+\Lambda\left(\frac{k+0.7 \pi}{M_{1}}\right)\right]
\end{aligned}
$$


according to the previous section, we have to choose $M_{1}$ such as

$$
\begin{aligned}
& \left\|F_{2}-F_{1}\right\|=\| 2 \operatorname{sinc}(0.7 \pi)\left[\Lambda\left(\frac{k-0.7 \pi}{M_{1}}\right)+\right. \\
& \left.+\Lambda\left(\frac{k+0.7 \pi}{M_{1}}\right)\right] \| \leq \frac{1}{K}
\end{aligned}
$$

On condition that the supports of the correcting roof functions do not superpose to each other, it can be seen that the norm at the left-hand side of inequality (4.4) is equal to $2 \sqrt{M_{1} / 3} \operatorname{sinc}(0.7 \pi)$, and therefore relationship (4.4), joined to the condition that the supports of the roof functions do not superpose to each other, provides the condition

$$
M_{1} \leq \min \left(\frac{3}{4 K^{2} \operatorname{sinc}^{2}(0.7 \pi)}, 0.7 \pi\right)
$$

In the case at hand, the second member of (4.5) is equal to $0.7 \pi$, so we can choose $M_{1}=0.7 \pi$.

At this point, the Inverse Fourier Transform of $F_{2}$ provides
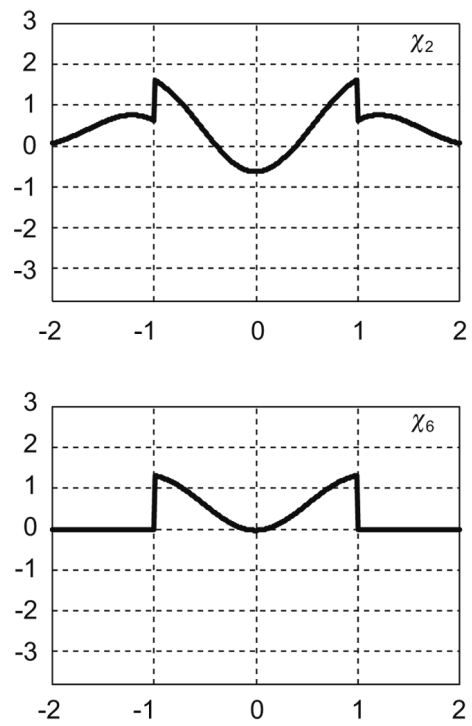

$\chi_{2}=\Pi\left(\frac{z}{2}\right)-4 M_{1} \operatorname{sinc}(0.7 \pi) \operatorname{sinc}^{2}\left(\frac{M_{1} z}{2}\right) \cos (0.7 \pi z)$.

And the truncation in spatial domain provides

$$
\begin{aligned}
& \chi_{2}=\Pi\left(\frac{z}{2}\right)-\Pi\left(\frac{z}{2}\right) 4 M_{1} \operatorname{sinc}(0.7 \pi) \operatorname{sinc}^{2}\left(\frac{M_{1} z}{2}\right) . \\
& \cdot \cos (0.7 \pi z)
\end{aligned}
$$

The Fourier Transform of $\chi_{3}$ provides

$$
\begin{aligned}
& F_{3}(k)=2 \operatorname{sinc}(k)-4 \operatorname{sinc}(k) \otimes \operatorname{sinc}(0.7 \pi) \\
& {\left[\Lambda\left(\frac{k-0.7 \pi}{M_{1}}\right)+\Lambda\left(\frac{k+0.7 \pi}{M_{1}}\right)\right]}
\end{aligned}
$$

where the symbol $\otimes$ stands for convolution product. At this point, $F_{4}$ is given by

$$
\begin{aligned}
& F_{4}(k)=F_{3}(k)-F_{3}(0.7 \pi)\left[\Lambda\left(\frac{k-0.7 \pi}{M_{2}}\right)+\right. \\
& \left.+\Lambda\left(\frac{k+0.7 \pi}{M_{2}}\right)\right] .
\end{aligned}
$$
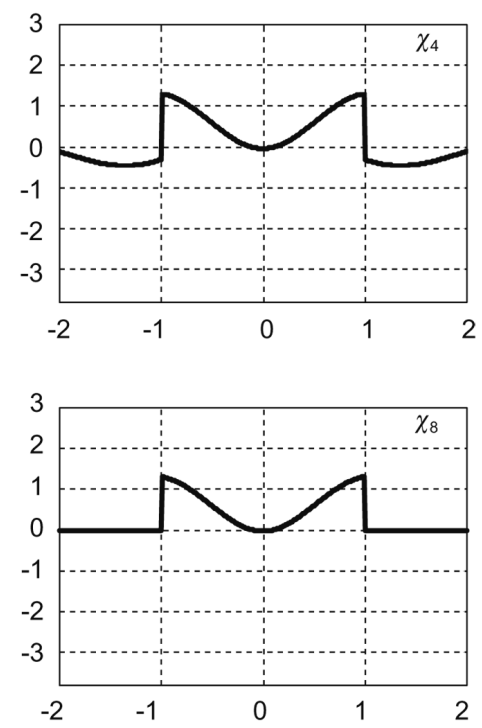

Fig. 2. Graphs of $\chi_{2}, \chi_{4}, \chi_{6}$ and $\chi_{8}$, dimensionless units. 

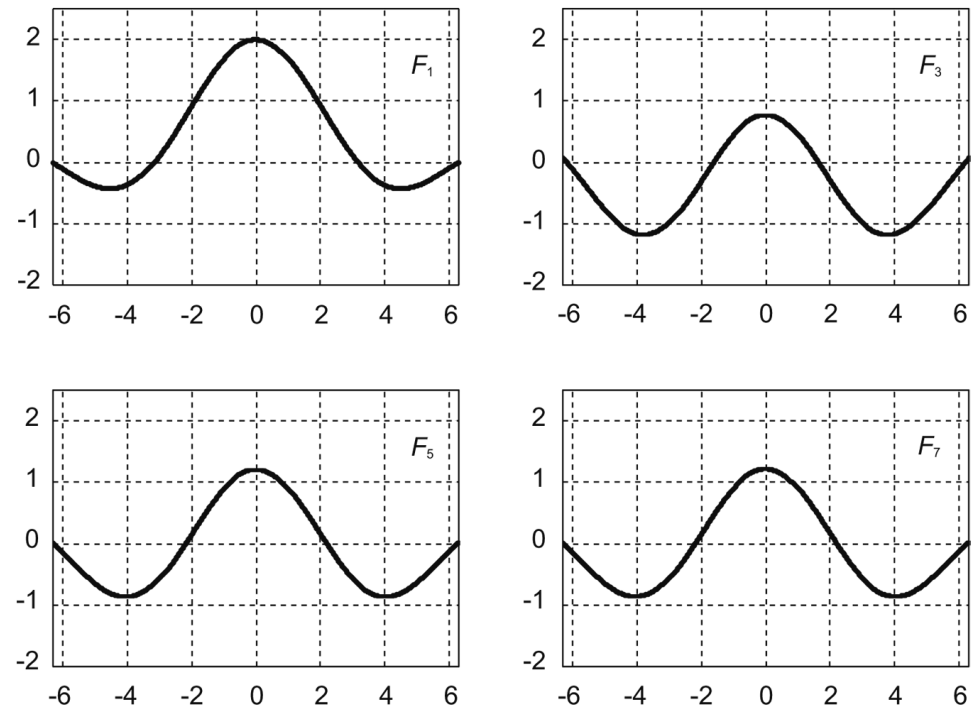

Fig. 3. Graphs of $F_{1}, F_{3}, F_{5}$ and $F_{7}$, dimensionless units.

And $M_{2}$ has to guarantee that

$$
\begin{aligned}
& \left\|F_{4}-F_{3}\right\|=\| F_{3}(0.7 \pi)\left[\Lambda\left(\frac{k-0.7 \pi}{M_{2}}\right)+\right. \\
& \left.+\Lambda\left(\frac{k+0.7 \pi}{M_{2}}\right)\right] \|<\frac{1}{K^{2}} .
\end{aligned}
$$

Inequality (4.10) can be solved essentially in the same way of inequality (4.4). Therefore, the condition on $M_{2}$ is still given by relationship (4.5) on condition that we change $K$ in $K^{2}$ and $4 \operatorname{sinc}^{2}(0.7 \pi)$ in $\left|F_{3}(0.7 \pi)\right|$. For this numerical example, the «compatible» value $M_{2}=0.4 \pi$ has been chosen. The procedure, which at this point is straightforward, has been iterated up to $\chi_{8}$ and $F_{8}$ (the subsequent compatible values $M_{3}=0.2 \pi$ and $M_{4}=0.1 \pi$ have been chosen).

Figure 2 represents the first four $\chi_{n}$ functions with even index (those with odd index are truncated, therefore they automatically verify the required condition about the support of the searched function). It can be seen that $\chi_{4}$ and $\chi_{6}$ are indistinguishable from a function supported in the interval $(-1,1)$.

Figure 3 represents the first four $F_{n}$ functions with odd index (those with even index are «corrected», therefore they automatically show the two required roots $\pm 0.7 \pi$ ). It can be seen that the first roots of $F_{5}$ and $F_{7}$ occur very near to the required points $\pm 0.7 \pi \cong \pm 2.199$.

\section{Conclusions}

This paper has shown a new proof of the non-uniqueness of the solution of the problem of the reconstruction of a compact supported function from a finite number of samples of its spectrum. This problem is, in particular, related to Diffraction Tomography, as shown in Section 2.

The problem has been formally afforded in $1 \mathrm{D}$ cases, but the extension to $2 \mathrm{D}$ or $3 \mathrm{D}$ cases is straightforward. In fact, the isometric properties of the Fourier transform hold also in $\mathrm{N}$-dimensional cases (for any integer $N$ ), as well as the triangular inequalities and all the mathematical statements exploited in Section 3. The only difference is that in an $\mathrm{N}$-dimensional case we should perform the corrections in the spectral domain by making use of some $\mathrm{N}$-dimensional version of the roof function (which can be provided by the product between $N$ one-dimen- 
sional roof functions versus each of the spectral variables).

A further development thought of is the extension of the proof presented here to the case of a finite number of samples of the Laplace Transform rather than the Fourier Transform. This extension is not straightforward for several reasons (e.g., some isometric properties of the Fourier Transform do not hold any longer for the Laplace Transform). However, such an effort seems worth trying, because it would also allow lossy cases to be considered in a rigorous way (Lesselier and Duchene, 1996).

\section{REFERENCES}

Alberti, G., L. Ciofaniello, M. Della Noce, S. Esposito, G. Galiero, R. Persico, M. Sacchettino and S. VeTRELLA (2002): A stepped frequency GPR system for underground prospecting, Ann. Geophysics, 45 (2), 375391.

Alberti, G., L. Ciofaniello, G. Galiero, R. Persico, M. Sacchettino, G.M. Signore and S. Vetrella (2003): Experimental results from a stepped frequency GPR, Ann. Geophysics, 46 (4), 707-717.

BERTERO, M. and P. BOCCACCI (1998): Introduction to Inverse Problems in Imaging (Taylor \& Francis), pp. 352.

Catapano, I., L. Crocco, R. Persico, M. Pieraccini and F. SOLDOVIERI (2006): Linear and non-linear microwave tomography approaches for subsurface prospecting: validation on real data, IEEE Trans. Antennas Wireless Propag. Lett., 5, 49-53.

CHEW, W.C. (1995): Waves and Fields in Inhomogeneous Media (IEEE Press, Piscataway, N.J.), pp. 608.

Clemmow, P.C. (1996): The Plane Wave Spectrum Representation of Electromagnetic Field (Wiley-IEEE Press), pp. 198.

Colton, D. and L. Paivarinta (1992): The uniqueness of a solution to an inverse scattering problem for electromagnetic waves, Arch. Rational Mech. Anal., 119, 59-70.

Crocco, L. and F. Soldovieri (2003): GPR prospecting in a layered medium via microwave tomography, Ann. Geophysics, 46, 559-572.

DANIELS, D. (2004): Ground Penetrating Radar (Inspec/Iee), 2nd edition, pp. 734.

Devaney, A.J. (1978): Nonuniqueness in the inverse scattering problem, J. Math. Phys., 19, 1526-1531.

FISHER, M. and K.J. LANGENBERG (1984): Limitations and defects of certain inverse scattering theories, IEEE Trans. Antennas Propag., AP-32, 1080-1088.

HANSEN, T.B. and P.M. JohANSEN (2000): Inversion scheme for ground penetrating radar that takes into account the planar air-soil interface, IEEE Trans. Geosci. Remote Sensing, 38, 496-506.

Kantorovic, L.V. and G.P. Akilov (1982): Functional Analysis (Pergamon Press), 2nd edition, pp. 589.

LeONE, G. and F. Soldovieri (2003): Analysis of the dis- torted Born approximation for subsurface reconstruction: truncation and uncertainties effects, IEEE Trans. Geosci. Remote Sensing, 41, 66-74.

LESSELIER, D. and B. DuChENE (1996): Wavefield inversion of objects in stratified environments: from back-propagation schemes to full solutions, in Review of Radio Science 1993-1996, edited by R. STONE (Oxford University Press, Oxford), 235-268.

Levin, B. (1980): Distribution of Zeros of Entire Functions (Providence, R.I., Am. Math. Soc.), 2nd edition, pp. 523.

LeVinson, N. (1940): Gap and Density Theorems (Am. Math. Soc.), pp. 246.

MeINCKE, P. (2001): Linear GPR inversion for lossy soil and a planar air-soil interface, IEEE Trans. Geosci. Remote Sensing, 39, 2713-2721.

PAley, R. and N. Wiener (1934): Fourier Transform in the Complex Domain (Am. Math. Soc., New York)).

Persico, R. and F. SOldovieri (2004a): Reconstruction of a slab embedded in a three layered medium from multifrequency data under Born approximation, J. Optical Soc. Am. A, 21, 35-45.

Persico, R. and F. Soldovieri (2004b): Effects of uncertainty on background permittivity in one dimensional linear inverse scattering, J. Optical Soc. Am. A, 21, 2334-2343.

Persico, R., R. Bernini and F. Soldovieri (2005): On the configuration of the measurements in inverse scattering from buried objects under the distorted Born approximation, IEEE Trans. Antennas Propag., 53, 1875-1886.

Persico, R., F. Soldovieri and G. Leone (2006): A microwave tomographic approach for multibistatic configuration: the choice of the frequency step, IEEE Trans. Instrum. Meas., 55 (6), 1926-1934, doi: 10.1109/ TIM.2006.884346.

PIERRI, R., R. PERSICO and R. BERNINI (1999): Information content of Born field scattered by an embedded slab: multifrequency, multiview, and multivifrequency-multiview cases, J. Optical Soc. Am. A, 16, 2392-2399.

RAMm, A.G. (1990): Completeness of the products of solutions of PDE and inverse problems, Inverse Problems, 6, 643-664.

SheEN, D. and D. ShePElsky (2000): Uniqueness in a frequency-domain inverse problem of a stratified uniaxial bianisotropic medium, Wave Motion, 31, 371-385.

SlePian, D. and H.O. Pollack (1961): Prolate spheroidal wavefunctions, Fourier analysis and uncertainty I, Bell Syst. Tech. J., 40, 43-64.

SOldOVIERI, F. and R. PERSICO (2004): Reconstruction of an embedded slab with Born approximation from multifrequency data, IEEE Trans. Antennas Propag., 52, 2348-2356.

Soldovieri, F., R. Persico and G. LeONE (2005a): Effect of source and receiver radiation characteristics in subsurface prospecting within the DBA, Radio Science, 40, RS3006.

Soldovieri, F., R. Persico and G. Leone (2005b): Frequency diversity in a linear inversion algorithm for GPR prospecting, Subsurface Sensing Technol. Appl., 6, 25-42.

(received February 2, 2006; accepted September 22, 2006) 